\title{
FACTORES SOCIALES EN ALGUNAS DIFERENCIAS LINGÜISTICAS EN CASTILLA LA VIEJA *
}

\author{
Flora Klein \\ (Georgetown University, USA)
}

La distinta distribución social de rasgos lingüísticos característicos de una región determinada puede investigatse como medida del «regionalismo implícito», o solidaridad regional, de entes tradicionalmente integrantes de dicha región. Para ello hay que hacer abstracción de las diferentes normas autóctonas locales, y buscar en cada localidad no tanto la frecuencia absoluta del rasgo lingǘistico en cuestión, sino más bien la «medida de prestigio» que disfruta localmente, reflejada en su distribución relativa en los distintos grupos sociales. Como ejemplo se ofrecen resultados sociolinguísticos preliminares del empleo de $l e, l a, l o$ en tres de las provincias históricamente integrantes de Castilla la Vieja.

* Agtadezco la ayuda económica de The American Pbilosopbical Society y el interés y acertados consejos de Ana María Barrenechea, Jesús M. de Miguel, William Labov, Beatriz Lavandexa y Alonso Zamora Vicente. 
En 1975 Jiménez Blanco y colaboradores publicaron el estudio La conciencia regional en España. ${ }^{1}$ Como su título indica, se trata de una comparación de las distintas comunidades históticas de España (y de las ciudades de Madrid y Barcelona) en cuanto al grado de "conciencia tegional» que manifiestan. Por consiguiente, dicha comparación se basa en gran parte en la medida en que los interrogados de cada zona geográfica muestran actitudes o aspiraciones comúnmente relacionados con el «regionalismo», en el sentido sociopolítico de la palabra.

Precisamente en este momento -en el que, por tazones obvias, han de suscitar el máximo interés las actitudes explícitas de individualidad en cuanto a valores e intereses regionales- será también especialmente interesante indagar hasta qué punto estas entidades históticas dan muestras de identidad sincrónica más o menos implícita o inconsciente, respecto a variables que normalmente no se relacionan con el «tegionalismo" sociopolítico. Ello permitirá, entre otras cosas, constatar hasta qué punto los dos tipos de actitudes - por una parte, las aspiraciones sociopolíticas explícitas, y por otra las conductas y valores inconscientes- pueden condicionarse mutuamente.

Tradicionalmente, el parecido lingüístico se ha considerado sintomático de identidad social o «comunidad». Así, por ejemplo, el mencionado estudio de Jiménez Blanco et al. encuentra que uno de los factores más distintivos de las regiones relativamente «regionalistas» es su percepción de diferencia lingüística respecto a las otras regiones. Ahora bien, ya que dicho trabajo investiga precisamente actitudes relativamente conscientes, las diferencias lingǘsticas que se desprenden como factores distintivos son también las más obvias - las que se perciben como manifestaciones de «un idioma diferente». Pero si quetemos investigar el sentimiento de comunidad en un sentido más encubierto o inconsciente, deberemos buscar

1. José Jiménez Blanco et al., La conciencia regional en Espoña (Madrid: Centro de Investigaciones Sociologicas, 1977) . 
su reflejo precisamente en rasgos lingüísticos relativamente desapercibidos en el habla normal, pero que sin embargo forman parte del repertorio del hablante, en primer lugar, en función de sta pertenencia a una «comunidad» determinada e incluso de su solidaridad con la misma. ${ }^{2}$

Por ello, como posible aportación a estudios de esta índole en Es. paña, ofrecemos aquí algunos resultados sociolingüísticos iniciales basados en los primetos datos obtenidos en una investigación, actualmente en curso, de la lengua hablada en Castilla la Vieja. Hasta ahora hemos investigado en cuatro de las ptovincias tradicionalmente incluidas en esta región - Valladolid, Burgos, Soria y Logroño- y hemos efectuado un análisis sociolingǘstico preliminar de muestras aleatorias del material obtenido en tres: Valladolid, Soria y Logroño.

\section{CuAdro 1}

Distintos sistemas de empleo de los clíticos le, la, lo $\mathrm{y}$ algunos de los empleos individuales consiguientes

\begin{tabular}{|c|c|c|c|}
\hline & $\begin{array}{c}\text { (A) } \\
\text { Sistema } \ll \text { Castal })\end{array}$ & $\begin{array}{c}(\mathrm{B}) \\
\text { Sistema } \\
\text { \&Referencial» }\end{array}$ & $\begin{array}{c}\text { Denominación } \\
\text { tradicional } \\
\text { del empleoreferencid }\end{array}$ \\
\hline i & $\begin{array}{l}\text { Lo conocí en la mili } \\
\qquad L a \text { conocí e }\end{array}$ & $\begin{array}{l}\text { Le conocí en ila mili } \\
\text { una fiesta }\end{array}$ & $\begin{array}{l}\text { «leísmo», zeferido a } \\
\text { un ser viviente }\end{array}$ \\
\hline iii & $\begin{array}{l}\text { Lo leímos en la escuela } \\
\qquad \text { Le dieron }\end{array}$ & $\begin{array}{l}\text { Le leímos en la escuela } \\
\text { cargo oficial }\end{array}$ & $\begin{array}{l}\text { «leísmo», teferido a } \\
\text { un ente inanimado }\end{array}$ \\
\hline $\begin{array}{l}\mathrm{v} \\
\mathrm{vi}\end{array}$ & $\begin{array}{l}\text { Su novio le dio una sortija } \\
\qquad \text { El vino to }\end{array}$ & $\begin{array}{l}\text { Su novio la dio una sortija } \\
\text { mamos con las comidas }\end{array}$ & «laísmo» \\
\hline vii & Al vino $l e$ echan de todo & Al vino lo echan de todo & «loísmo» \\
\hline
\end{tabular}

2. Quizás el ejemplo ciásico del efecto del factor «solidaridad» en la adopción de rasgos linguísticos locales sea el estudio de William Labov «The Social Motivation of a Sound Changes, Word 19 (1963): 273.309, reproducido en Sociolinguistic Patterns del mismo autor (Philadelphia: University of Pennsylvania Press, 1972), cap 1. 


\section{Cuadro 2}

Frecuencia de empleos referenciales en varones de Valladolid, de Soria y de Logroño

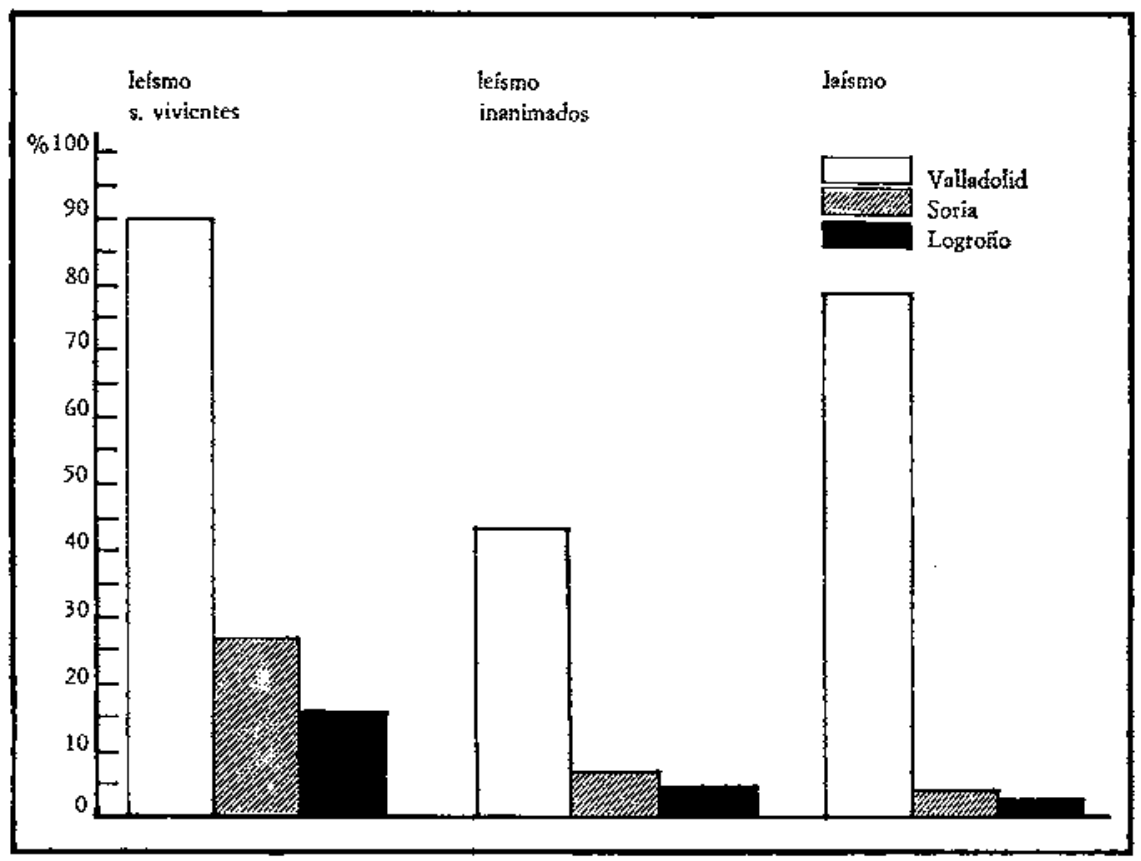

\begin{tabular}{|c|c|c|c|c|c|c|c|c|c|}
\hline \multirow[b]{2}{*}{ Provincias: } & \multicolumn{3}{|c|}{$\begin{array}{c}\text { Leismo } \\
\text { seres vivientes }\end{array}$} & \multicolumn{3}{|c|}{$\begin{array}{c}\text { Leismo } \\
\text { inanimados }\end{array}$} & \multicolumn{3}{|c|}{ Lásmo } \\
\hline & $\begin{array}{l}\text { Núm. } \\
\text { total }\end{array}$ & $\underset{\text { Núm. }}{\text { le }}$ & $\begin{array}{l}\% \\
\mathrm{le}\end{array}$ & $\begin{array}{l}\text { Núm. } \\
\text { total }\end{array}$ & $\begin{array}{c}\text { Núm. } \\
\text { le }\end{array}$ & $\begin{array}{c}\% \\
\text { le }\end{array}$ & $\begin{array}{l}\text { Núm. } \\
\text { total }\end{array}$ & Núm. & $\begin{array}{c}\% \\
\mathrm{I} a\end{array}$ \\
\hline Valladolid & 195 & 175 & $90 \%$ & 117 & 53 & $45 \%$ & 58 & 46 & $79 \%$ \\
\hline Soria & 148 & 40 & $27 \%$ & 119 & 8 & $7 \%$ & 125 & 5 & $4 \%$ \\
\hline Logroño & 103 & 16 & $16 \%$ & 105 & 5 & $5 \%$ & 45 & 1 & $2 \%$ \\
\hline
\end{tabular}


E1 rasgo especiffico que investigamos es el uso de los clíticos $l e$, la y lo. Se ha observado repetidamente que Castilla difiete del testo del mundo hispanohablante, y por lo tanto del testo de España, en su modo de usar estos elementos. Más concretamente, se ha observado que el concepto lingǘstico denominado «caso» (que a grandes rasgos parece referirse a la relativa aportación del ente en cuestión a la actividad o situación que designa el verbo) ${ }^{3}$ es relativamente irrelevante al uso de los clíticos en Castilla, el cual parece basarse más bien en características referenciales descriptivas) del ente en sí: por ejemplo, su sexo, si se trata de un ser viviente, o si se trata de un ente inanimado (una cosa, una abstracción, etc.), su género gramatical ${ }^{4}$ y su grado relativo de individualización. ${ }^{5}$ Este sistema de empleo de $l e, l a$ y $l o$, que podemos denominar «acasual» - «referencial», da lugat a que sean corrientes en Castilla usos como los ejemplificados bajo (B) en el Cuadro 1. Sus contrapartidas en el resto del mundo hispanohablante monde el empleo de $l e, l a$ y $l o$ parece basarse en primer lugar en la distinción de caso, por lo que podemos llamarlo «casual»- son las que aparecen bajo (A). ${ }^{6}$

Aqui debemos notar, antes que nada, que las observaciones anteriores sobre esta cuestión se basaron principalmente en impresiones, apoyadas de algunas estadísticas basadas en la lengua escrita -especialmente el uso literario.? Así que no es de extrañar que el primer resultado que surgió de la presente investigación, basada en extensas grabaciones de la lengua hablada, fue el constatar que el empleo de los clíticos no es uniforme ni siquiera en las cuatro provincias investigadas, tradicionalmente integtantes de Castilla la Vieja. Así encontramos que el empleo «teferencial» considerado típico de Castilla [ejemplificado bajo (B) en el Cuadro 1] predomina

3. Véase, por ejemplo, Erica C. Garcia, The Role Theory in Linguistic Analysis: The Spanish Pronoun System (Amsterdam: North Holland, 1975).

4. Véase, por ejemplo, Rafael Lapesa, Historia de la Lengua Española, 4a. ed. (Nueva York: Las Américas, 1959).

5. La importancia de esta característica referencial solo parece haber sido observada hasta ahora en hablas geográfica y socialmente restringidas: Ralph J. Penny, El babla pasiega (Londres: Támesis, 1971); José Millán Urdiales, El babla de Villacidayo (León) (Madrid: Boletín de la Real Academia Española, Anexo XIII, 1966). Pero nuestra investigación indica que de hecho tiene mucha mayor extensión, tanto social como geográfica, aparentemente en toda la zona «referencial».

6. Los aspectos puramente lingǘísticos de esta diferencia (en particular, las bases para el análisis semántico de los dos sistemas) serán tratados en otro lugar.

7. Rufina José Cuervo, «Los casos enclíticos y procí́ticos del pronombre de tercera persona en casteliano», Romania XXIV (1895): 95-244; Salvador Fernández, Gramática española (Madrid: Revista de Occidente, 1950). 
Diferencias linguísticas en Castilla la Vieja

\section{Cuadro 3}

Frecuencia de empleos referenciates en varones de Valladolid en función de la clase social del hablante
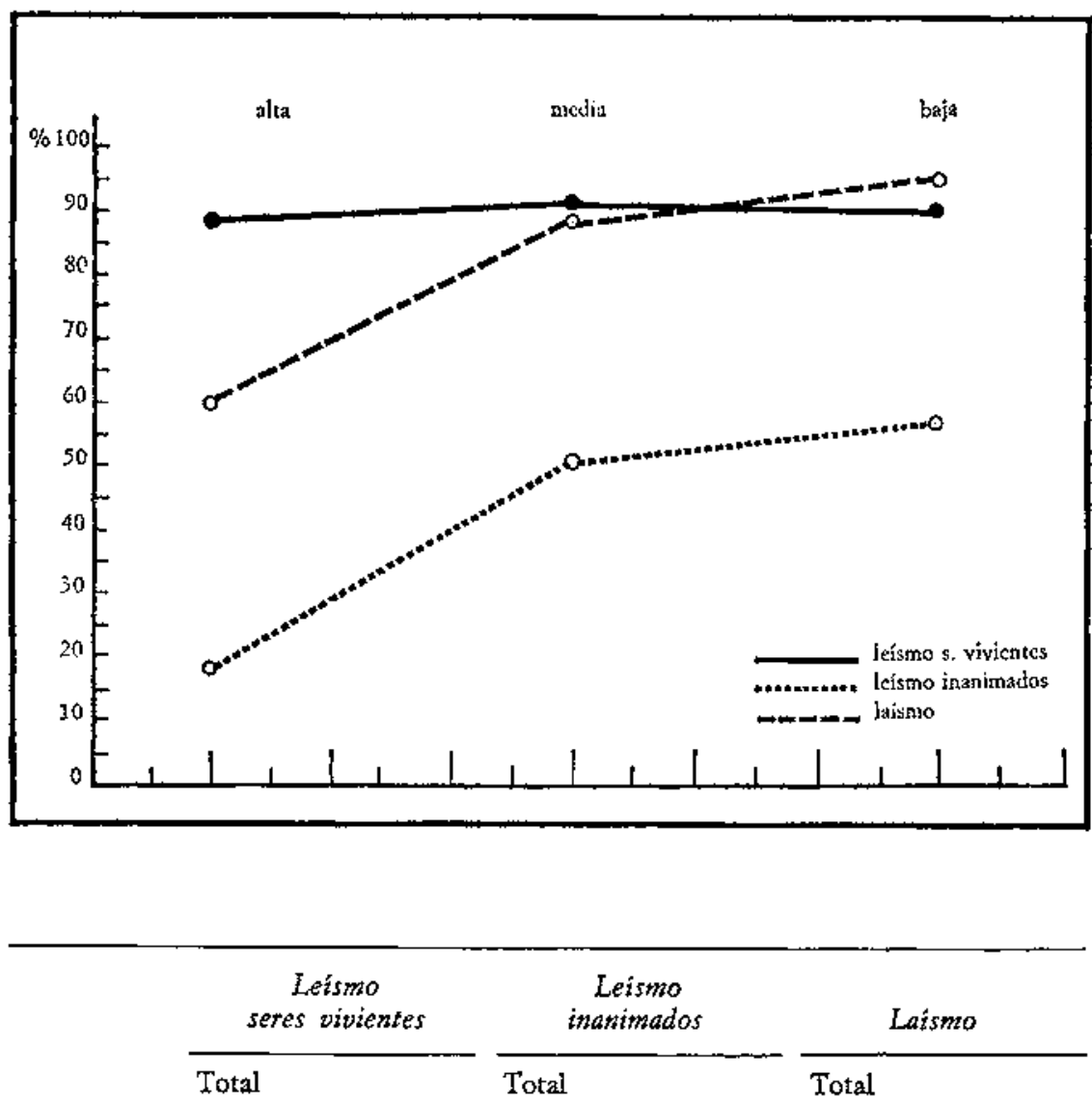

Clase social; núm. Núm.le \% le núm. Núm.le \%le núm. Núm. Ia \% la

\begin{tabular}{|c|c|c|c|c|c|c|c|c|c|}
\hline Alta & 34 & 30 & $88 \%$ & 28 & 5 & $18 \%$ & 22 & 13 & $59 \%$ \\
\hline Media & 108 & 93 & $90 \%$ & 38 & 19 & $50 \%$ & 18 & 16 & $89 \%$ \\
\hline Baja & 58 & 52 & $90 \%$ & 51 & 29 & $57 \%$ & 18 & 17 & $94 \%$ \\
\hline
\end{tabular}


«Papers»: Revista de Sociología

\section{CUADRO 4}

Frecuencia de usos teferenciales en Valladolid en función de clase y sexo del hablante

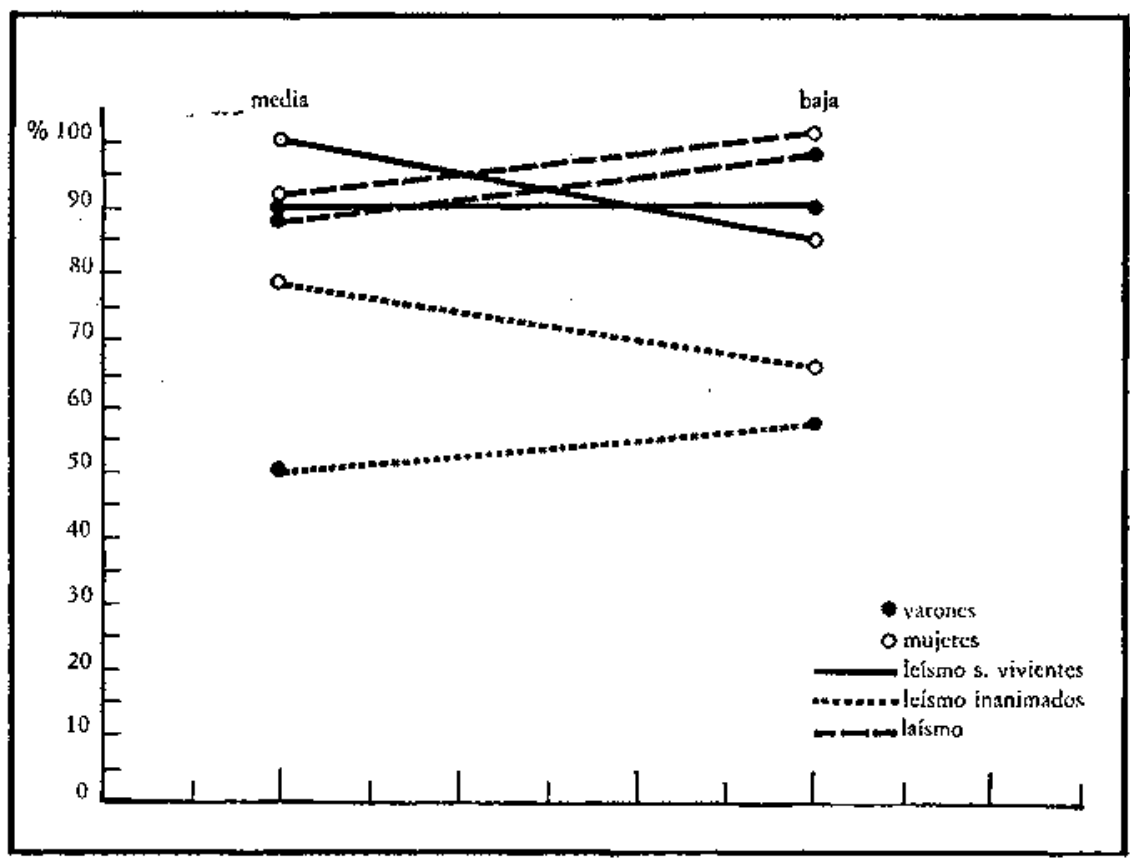

\begin{tabular}{|c|c|c|c|c|c|c|c|c|c|}
\hline & \multicolumn{3}{|c|}{$\begin{array}{c}\text { Leismo } \\
\text { seres vivientes }\end{array}$} & \multicolumn{3}{|c|}{$\begin{array}{c}\text { Leismo } \\
\text { inanimados }\end{array}$} & \multicolumn{3}{|c|}{ Laismo } \\
\hline & $\begin{array}{l}\text { Total } \\
\text { núm. }\end{array}$ & Núm. le & $\%$ ie & $\begin{array}{l}\text { Total } \\
\text { núm. }\end{array}$ & Nún. Je & $\%$ le & $\begin{array}{l}\text { Total } \\
\text { núm. }\end{array}$ & Núm. la & $\%$ ia \\
\hline \multicolumn{10}{|l|}{ Clase media: } \\
\hline Varones & 103 & 93 & $90 \%$ & 38 & 19 & $50 \%$ & 18 & 16 & $89 \%$ \\
\hline Mujeres & 21 & 21 & $100 \%$ & 60 & 47 & $78 \%$ & 33 & 30 & $91 \%$ \\
\hline \multicolumn{10}{|l|}{ Clase baja: } \\
\hline Varones & 58 & 52 & $90 \%$ & 51 & 29 & $57 \%$ & 18 & 17 & $94 \%$ \\
\hline Mujeres & 28 & 24 & $86 \%$ & 88 & 58 & $66 \%$ & 29 & 28 & $96 \%$ \\
\hline
\end{tabular}


sólo en las más occidentales de las cuatro provincias estudiadas: Burgos y Valladolid. En cambio, el empleo de los clíticos en las dos más orientales - Soria y Logroño- se parece más al del resto del mundo hispanohablante, en cuanto responde en primer lugar a distinciones de caso [como el ejem. plificado más abajo (A)].

Hasta qué punto difiere la norma hablada de estas dos zonas de Castilla podrá apreciarse en el Cuadro 2, donde se compara la incidencia en el habla de varones de Valladolid, de Soria y de Logroño de usos «referenciales» en los contextos que se dan con mayor frecuencia en el discurso. - los que dan lugar a los ejemplos (i) (iii) y (iv) del Cuadro 1 y, por lo

\section{Cuadro 5 (a)}

Lugar de origen de los interrogados y número de interrogados de cada lugar

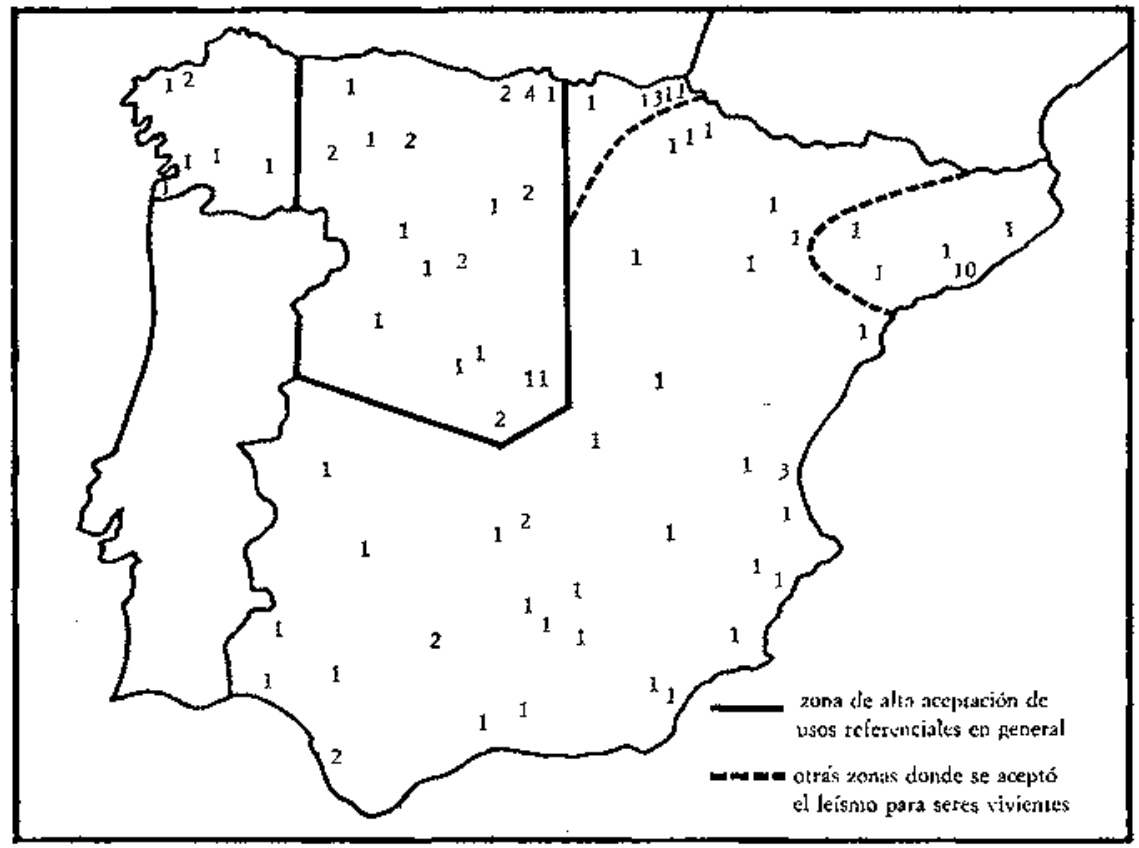

8. Puesto que los contextos son semánticos su relativa frecuencia en el discurso depende del tema de que se hable. Aquí nos referimos a los temas que surgieron en las entrevistas iniciales más o menos espontáneamente. 
tanto, a los empleos referenciales comúnmente denominados «leísmo» y «laísmo».

Ahora bien: la Sociolingüística ha demostrado que el indicio linguístico que muestra mejor correspondencia con entidades delimitadas a priori por criterios geopolíticos o históricos (el caso de las regiones españolas, así como de ciudades, etc.) no es tanto su homogeneidad en el comportamiento linguístico mismo, el cual típicamente varía en función de factores sociales, demográficos y estilísticos. En entidades de este tipo el mejor indicio de «comunidad» parece ser más bien el parecido en la evaluación social, positiva o negativa, que comportan determinados rasgos lingüísticos."

Cuadro 5 (b)

Aceptación de distintos usos referenciales en función del origen geográfico del interrogado

\begin{tabular}{|c|c|c|c|c|c|c|c|c|c|}
\hline & \multirow{2}{*}{$\begin{array}{c}\text { Núm. } \\
\text { total } \\
\text { interro- } \\
\text { gados }\end{array}$} & \multicolumn{2}{|c|}{$\begin{array}{l}\text { Aceptacion de } \\
\text { leismo para } \\
\text { seres vivientes }\end{array}$} & \multicolumn{2}{|c|}{$\begin{array}{l}\text { Aceptación de } \\
\text { letsmo para } \\
\text { inanimados }\end{array}$} & \multicolumn{2}{|c|}{$\begin{array}{c}\text { Aceptacion } \\
\text { de laismo }\end{array}$} & \multicolumn{2}{|c|}{$\begin{array}{l}\text { Aceptacion } \\
\text { de loismo }\end{array}$} \\
\hline & & Núm. & $\%$ & Núm. & $\%$ & Núm. & $\%$ & Núm. & $\%$ \\
\hline $\begin{array}{l}\text { Dentro de la zona } \\
\text { delimitada en (5a } \\
\text { por la línea negra }\end{array}$ & $\begin{array}{ll}\text { a } & 34 \\
\text { a) } & \\
\text { a } & \\
& 34\end{array}$ & 27 & $79 \%$ & 10 & $29 \%$ & 20 & $59 \%$ & 5 & $15 \%$ \\
\hline continua: & 34 & 27 & $79 \%$ & 10 & $29 \%$ & 20 & $59 \%$ & 5 & $15 \%$ \\
\hline $\begin{array}{l}\text { En el resto de la } \\
\text { península: }\end{array}$ & 68 & 28 & $41 \%$ & 5 & $7 \%$ & 3 & $4 \%$ & 0 & $0 \%$ \\
\hline
\end{tabular}

De hecho, la evaluación social que comporta un tasgo lingüístico $\mathrm{X}$ en una localidad determinada puede captarse en diversas medidas, una de las cuales es precisamente la distribución del rasgo $\mathrm{X}$ en el habla de distintos grupos sociales y demográficos locales. Como se ha constatado repetidamente, en la situación relativamente intimidante de una entrevista grabada ciertos grupos tienden más que otros a adoptar los rasgos valorados posi-

9. William Labov, Sociolinguistic Patterns (Philadelphia: University of Pennsylvania Press, 1972), capítulo 6. 
Diferencias lingüisticas en Castilla la Vieja

Cuadro 6

Frecuencia de empleos referenciales en varones de Soria en función de clase social

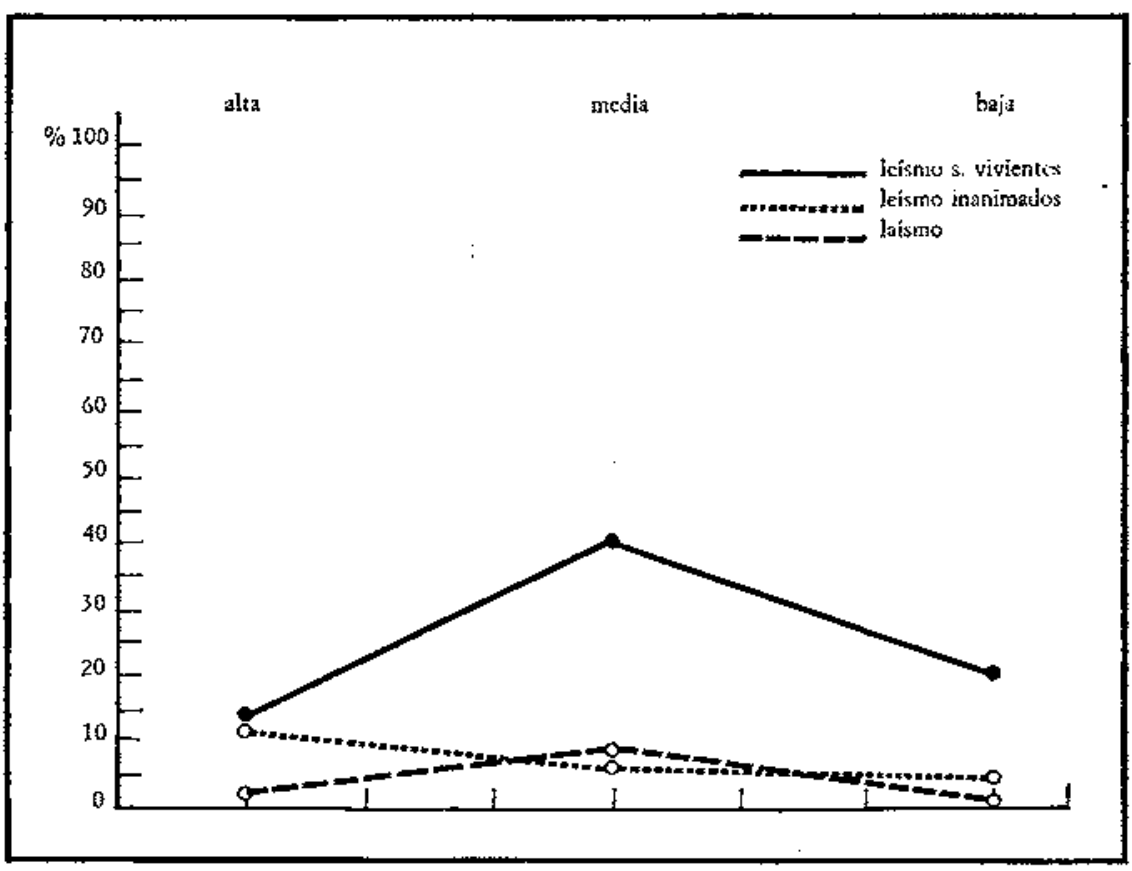

\begin{tabular}{|c|c|c|c|c|c|c|c|c|c|}
\hline \multirow[b]{2}{*}{ Clase social: } & \multicolumn{3}{|c|}{$\begin{array}{c}\text { Leismo } \\
\text { seres vivientes }\end{array}$} & \multicolumn{3}{|c|}{$\begin{array}{c}\text { Leismo } \\
\text { inanimados }\end{array}$} & \multicolumn{3}{|c|}{ Laismo } \\
\hline & $\begin{array}{l}\text { Total } \\
\text { núm. }\end{array}$ & Núm. le & $\%$ le & $\begin{array}{l}\text { Total } \\
\text { núm. }\end{array}$ & Núm. Ie & $\%$ le & $\begin{array}{l}\text { Total } \\
\text { núm. }\end{array}$ & Núm. la & $\%$ ia \\
\hline Alta & 29 & 4 & $14 \%$ & 27 & 3 & $11 \%$ & 45 & 1 & $2 \%$ \\
\hline Media & 63 & 25 & $40 \%$ & 65 & 4 & $6 \%$ & 61 & 4 & $7 \%$ \\
\hline Baja & 56 & 11 & $20 \%$ & 27 & 1 & $4 \%$ & 19 & 0 & $0 \%$ \\
\hline
\end{tabular}


tivamente en la localidad, evitando los de valoración local negativa - siendo ambas actitudes verificables por medidas independientes, más directas. Esta tendencia a «autocorregirse» en las direcciones más aceptadas localmente ha demostrado ser típica de hablantes de clase media (y especialmente de clase media baja), así como también de mujeres..$^{10}$ Por consiguiente, en principio las tendencias lingǘsticas registradas en estos dos grupos de ha. blantes podrán considerarse como probables indicadores de valores sociolingüísticos locales.

Teniendo esto en cuenta podemos realizar una prueba inicial del grado de cohetencia sociolingǘrstica que puedan manifestar las provincias castellanas de Valladolid, Soria y Logroño, respecto a la variable investigada: el empleo de le, la y $l o$.

Veamos en primer lugar la distribución de usos referenciales (de nuevo, sólo los tres de mayor incidencia en el discurso) en Valladolid, en función de clase social en el Cuadro 3 y de clase social y sexo en el Cuadro $4 .^{11}$ Los resultados indican, primero, que en esta provincia el uso autóctono o nativo es el referencial, puesto que éste es típico de los hablantes de clase baja, los cuales en este caso son todos rurales. Es más, en Valladolid el uso referencial parece constituir la norma bablada o vernácula, ya que vemos que también caracteriza el habla de la clase media -.-varones y mujeres, aunque en esta clase social aún más la de las mujeres. En Valladolid, pues, sólo la clase alta (que en esta primera muestra se compone sólo de varones) tegistra frecuencias relativamente más bajas de empleos referenciales -en general. Pero es significativo que esta tendencia no se extiende a uno de los usos referenciales: el leismo teferido a seres vivientes. Como se ve en el Cuadro 3, la frecuencia de este empleo es prácticamente la misma en vallisoletanos de las tres clases sociales.

Los resultados obtenidos de los vallisoletanos de clase alta concuerdan con un hecho que pudimos constatar en una encuesta previa, basada en cuestionarios, los resultados de la cual se ven en el Cuadro 5..$^{12}$ Primero, encontramos que el empleo de los clíticos más aceptado en la península en

10. Ibidem, capítulos 5,8 y 9 .

11. Para los efectos del presente trabajo las clases sociales son: Alta $=$ altos ejecutivos o profesionales; Baja $=$ trabajadores manuales; Media $=$ los restantes: pequeños comerciantes, empleados white collar, y funcionarios sin especial prestigio a nivel provincial. En general, una clase social está representada en cada provincia por dos o tres hablantes de cada sexo, menos la clase alta que consta aquí de dos varones en Valladolid, dos varones en Soria y una mujer y un varón en Logroño.

12. A los entrevistados les fueron presentadas dos alternativas (por escrito en general, saivo en el caso de alguna persona analfabeta a quien le fueron leídas) como las del Cuadro 1 , una del tipo (A) y otra del (B). Se les pidió que marcaran la que les 
general es el que se basa en distinción de caso - el que se ejemplifica bajo (A) en el Cuadro 1. Pero también encontramos que los distintos empleos referenciales [bajo (B)] gozan de muy distinto grado de aceptación -tanto dentro como fuera de la zona que se distinguió por su mayor aceptación de empleos refexenciales en general (la delimitada por la línea negrt continua en el Cuadro 5a). Concretamente vemos que el empleo referencia! más aceptado - dentro y fueta de esta zona, pero naturalmente no en el mismo grado en las dos condiciones- es precisamente el lésmo referido a seres vivientes. ${ }^{13}$ Ahora bien: la mayor aceptación de leísmo para seres vivientes, comparado con los otros empleos referenciales, se manifiesta no sólo en un sentido geogrático, sino que también (es de suponer que en consecuencia de ello) en un sentido estilístico. Así vemos que el leísmo para seres vivientes es ef único de los empleos referenciales que se acepta en la lengua escrita, así como en otros usos típicamente «normatizantes» como son el lenguaje de conferencias, el de locutores de radio y televisión, etc. ${ }^{14}$ Así que pođemos decir que el habla de los vallisoletanos de clase. alta se acerca relativamente más a la norma de aceptación más general

parecía más corriente en su tierra (en el lugar donde figuran en 5a), dándoseles también la opción de marcar las dos si les parecían igualmente «normales». Los resultados presentados en el Cuadro 5 incluyen todos los casos de aceptación del empleo en cuestión, tanto los que excluían el empleo alternativo como los que lo aceptabart también.

13. E1 hecho de que al leismo para seres vivientes goce de mayor aceptación que los otros empleos xeferenciales ( $y$ que ai parecer haya sido así desde sus orígenes: véase Rafael Lapesa, «Sobre los orígenes y evolución del leísmo, laísmo y toísmo», pp. 523-551, en K. Baldinget, Festscbrift von Walter von Wartburg [Tubingen, 1968]) es interesante pero no particularmente extraño. En primer lugar, la evaluación social de rasgos linguísticos típicamente no es consecuente, en el sentido de que no se les atribuye necesariamente un mismo valor social (positivo o negativo) a todos los rasgos que, de un punto de vista jingüístico, pueden ser interpretados como manifestaciones de un mismo fenómeno (Wiliam Labov, 1972, op. cit., p. 181). Por otra parte, también hay motivos puramente lingüísticos para considerar que, en igualdad de condiciones, el leísmo para seres vivientes pueda ser telativamente más coherente con el mismo sistema casuat (A) que los otros usos referenciales (B), lo cual lo haría relativamente más aceptable a los usuarios de (A) (Erica García, 1975, op. cit., capítulo 7).

14. Como el empleo referencial en general es sólo un empleo vernáculo u oral (con la significativa excepción del leismo para seres vuvientes), los medios escritos o susceptibles de ser interpretados como «lengua escrita» serán aptopiados para investigarlo sólo de modo preliminar o accesorio. En particular, sospecharemos que los indicios de empleo referencial que puedan detectarse por tales medios pecarán de conservadores, en el sentido de que la aceptación de un empleo referencial X en una localidad L podtá interpretarse, en principio, como indicación de la existencia efectiva de $\mathrm{X}$ en el habla de $L$, pero no lo contratio: ta no aceptación de $X$ no indicará necesariamente su inexistencia. En efecto, nuestra actual investigación de la lengua hablada nos ha 
"Papers»: Revista de Sociologia

\section{Cujadro 7}

Frecuencia de empleos referenciales en Soria en función de la clase social y sexo del hablante

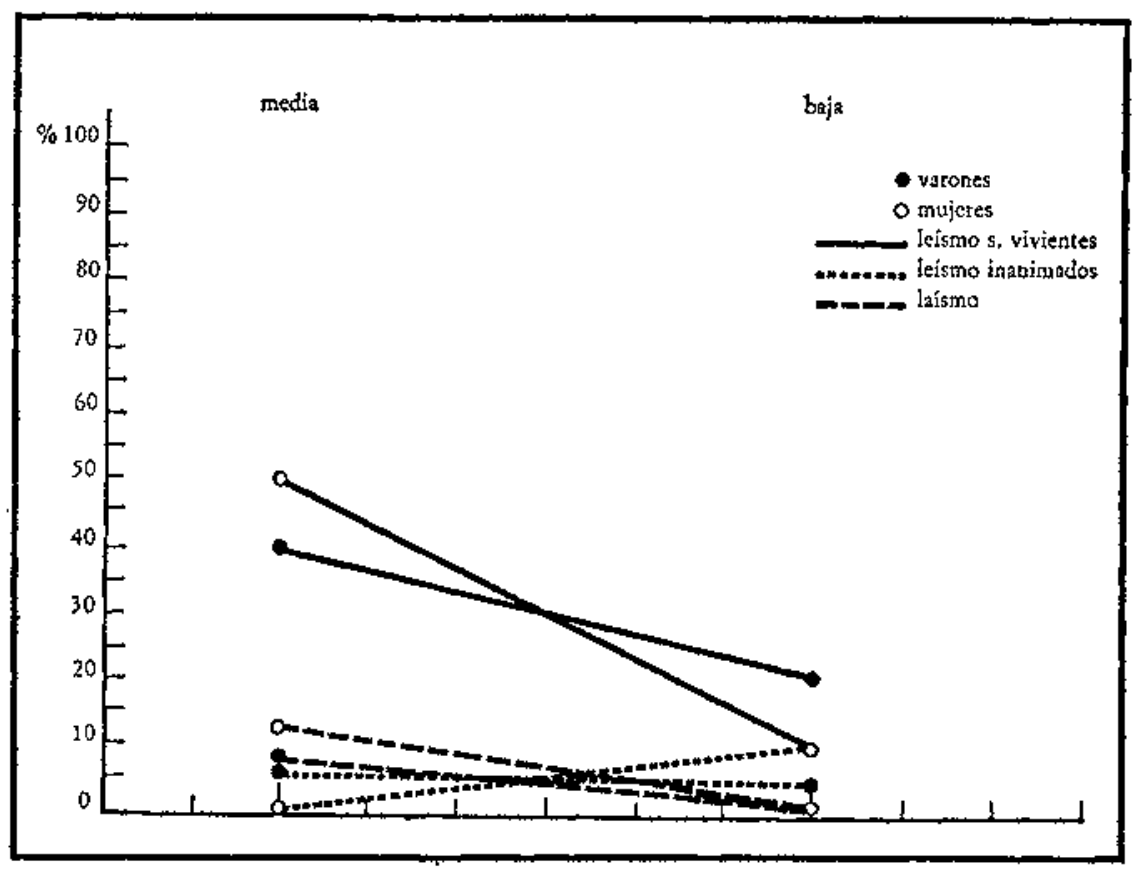

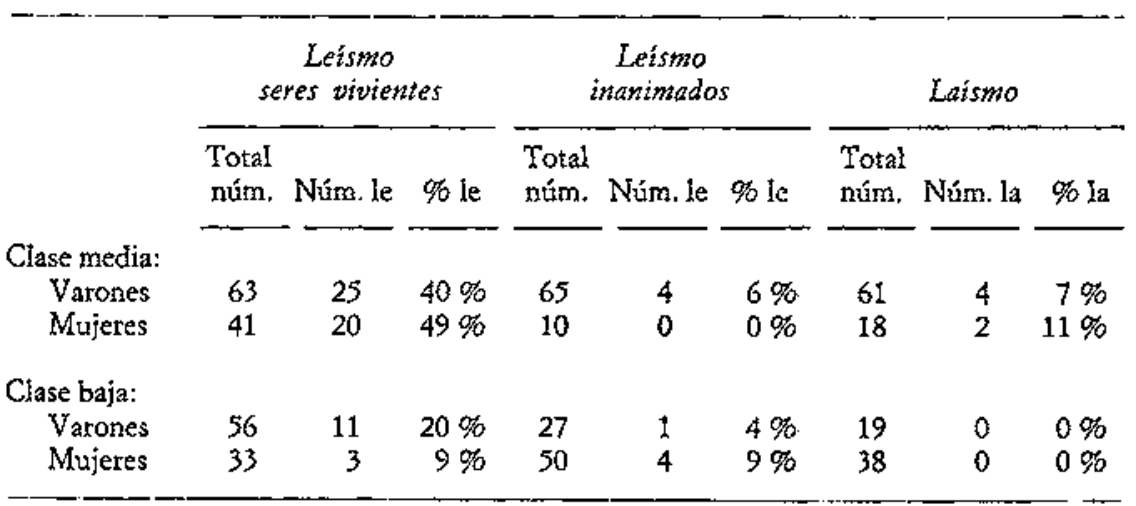


(y en particular a la de la lengua escrita) que la de los otros grupos locales.

Veamos ahora en los Cuadros 6 y 7 los resultados obtenidos en Soria. En primer Iugar se observa que en esta provincia la frecuencia de empleos referenciales es relativamente baja en los hablantes de clase baja. Puesto que aquí también los hablantes de clase baja fueron rurales, y nativos, podemos concluir que aquí el uso autóctono es el que se basa en distinción de caso. ${ }^{15}$ Asimismo encontramos baja la frecuencia de empleos referenciales entre sorianos de clase alta, por lo cual podemos concluir que éstos también mantienen el uso autóctono. Peto en cambio entre los sotianos de clase media la frecuencia de por lo menos uno de los usos tefetenciales - el leismo para setes vivientes- sube marcadamente, subiendo también la del laismo, aunque mucho menos. $Y$ estas tendencias se observan en varones y en mujeres, aunque algo más en mujeres. Ello sun giere que esta zona -aun siendo predominantemente «casual» en su empleo de los clíticos y en este sentido más parecida al mundo hispanohablante fuera de la misma Castilla- no obstante da muestras de valorar positivamente usos típicos de Castilla, en la tendencia a adoptar estos usos por parte de la clase media.

Si examinamos ahora los resultados obtenidos en Logroño, vemos primero en el Cuadro 8 que entre los varones riojanos la incidencia global de empleos referenciales es aún más baja que entre los sorianos. Y también vemos que en Logroño los hablantes de clase media difieren muy poco, en este aspecto, de los de clase baja. Por lo tanto, en lo que respecta al uso de los clíticos podríamos decir que de las tres provincias examinadas Logrono es la menos «castellana», usando la palabra en sentido sincrónico y geográfico.

Pero aún aquí la distribución social de usos referenciales indica que por lo menos uno de ellos goza localmente de prestigio: el leísmo para seres vivientes, que como ya se ha observado es el uso referencial que disfruta mayor aceptación en general. Así vemos en el Cuadro 8 que, entre los varones riojanos, este uso es notablemente más frecuente en la clase alta que en la media y la baja. Pero más significativo es el resultado que se

permitido constatar que el empleo teferencial tiene una extensión geográfuca más amplia que la delimitada en $5 a$.

15. Para mayor exactitud, esto es aplicable por lo menos a la misma capital y a los pattidos de Soria y de Agreda. En cambio, hay una zona de la provincia, que parece incluir por lo menos el partido occidental deł Burgo de Osma, donde el uso nativo es ya el referencial, a juzgar por el habla de la clase baja rural. Para evitar en lo posible el confundir factores geográficos con factores sociales, excluimos de nuestros datos de Soria los obtenidos de hablantes del partido del Burgo. 
«Papers»: Revista de Sociología

\section{CuAdro 8}

Frecuencia de empleos referenciales en varones de Logroño en función de la clase social

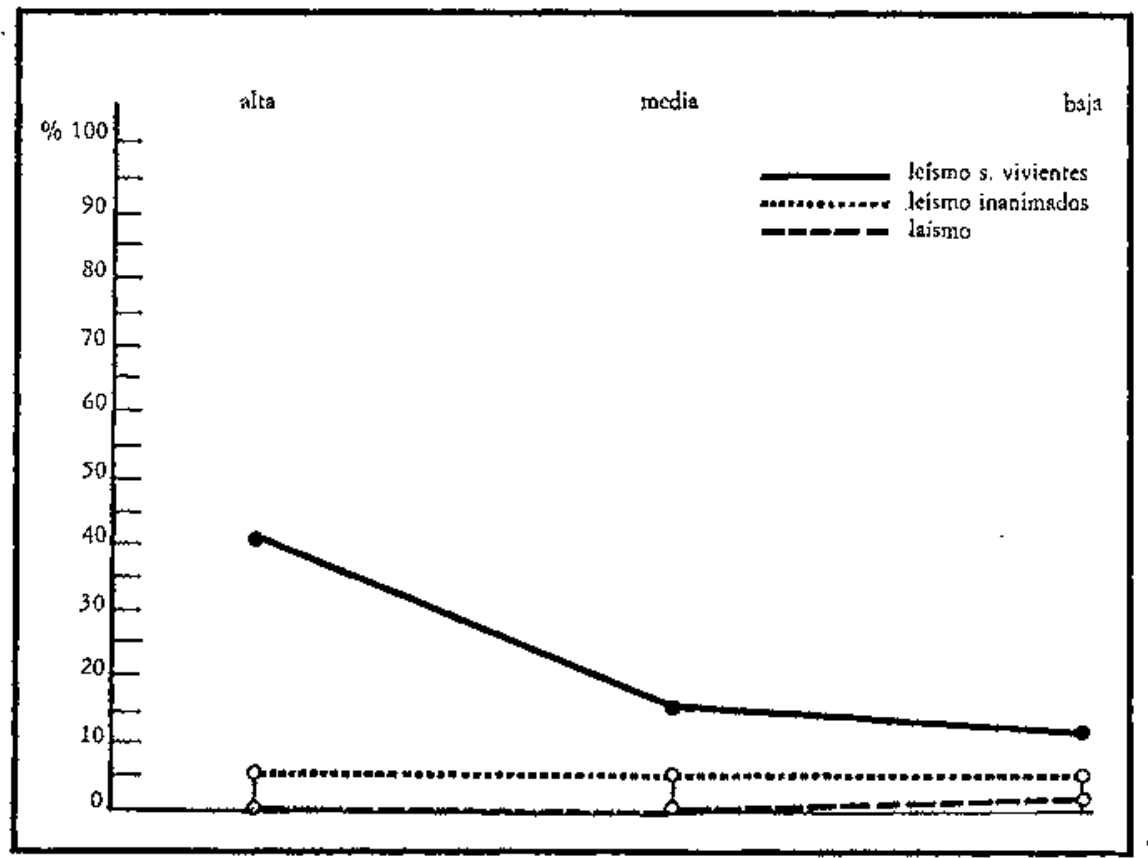

\begin{tabular}{|c|c|c|c|c|c|c|c|c|c|}
\hline \multirow[b]{2}{*}{ Clase social: } & \multicolumn{3}{|c|}{$\begin{array}{c}\text { Leismo } \\
\text { seres vivientes }\end{array}$} & \multicolumn{3}{|c|}{$\begin{array}{c}\text { Leîsmo } \\
\text { inanimados }\end{array}$} & \multicolumn{3}{|c|}{ Laismo } \\
\hline & $\begin{array}{l}\text { Total } \\
\text { núm. }\end{array}$ & Núm. le & $\%$ le & $\begin{array}{l}\text { Total } \\
\text { núm. }\end{array}$ & Núm. le & $\%$ le & $\begin{array}{l}\text { Total } \\
\text { núrn. }\end{array}$ & Núm. la & $\%$ la \\
\hline Alta & 13 & 5 & $38 \%$ & 20 & 1 & $5 \%$ & 6 & 0 & $0 \%$ \\
\hline Media & 36 & 5 & $14 \%$ & 44 & 2 & $5 \%$ & 16 & 0 & $0 \%$ \\
\hline Baja & 54 & 6 & $11 \%$ & 41 & 2 & $5 \%$ & 45 & 1 & $2 \%$ \\
\hline
\end{tabular}


observa en el Cuadro 9, donde vemos que la frecuencia de este empleo en Logroño es sistemáticamente mucho más alta entre mujeres que entre va* rones, manteniéndose esta diferencia entre los sexos en cada una de las clases sociates. Estos resultados indican, pues, que en Logroño por to menos esta manifestación particular del empleo referencial de Castilla se trata como rasgo prestigioso.

No bay que decir que estos resultados no bastarían, ni mucho menos, para apoyar ni desmentir posibles hipótesis respecto a identidad de valores implicitos en Castilla la Vieja -entre otras cosas porque no tenemos datos de todas las provincias tradicionalmente integrantes de esta tegión, ni tampoco (para efectos de comparación) de ninguna que no lo sea. No obstante, gtacias a la gran sistematicidad que muestra el idioma cuando se estudia como fenómeno social, sí permiten demostrar lo que podría aportar la sociolingüística a una investigación de esa índole. En este sentido lo más interesante no es tanto la envergadura de los datos ni la contundencia de los resultados, sino más bien su coherencio con principios y hallazgos socio. lingüísticos en general, y en particular con la situación y dinámica social de la zona investigada.

Recordemos que la teoría sociolingürstica, y la metodología que de ella se desprende, se basan precisamente en el supuesto de que las tenden* cias lingüísticas estarán condicionadas, entre otras cosas, por la estructura y la dinámica social. Y zecordemos también que los resultados socio-lingüisticos que mayor coherencia han mostrado con coordenadas sociales han sido precisamente las evaluaciones de rasgos lingǘsticos (tanto las reflejadas en medidas más o menos explícitamente evaluativas, como las impir. citas en la estratificación social o estilfstica del habla misma), y que uno de los resultados más reveladores al respecto ba sido precisamente la constatación de discrepancias, dentro de una misma localidad, entre el comportamiento lingüístico predominante y la valoración social del mismo.

Teniendo esto en cuenta se apreciará el interés que tienen, por ejemplo, los resultados obtenidos en Soria. Aquí lo interesante es que, a pesar de que el vernáculo autóctono (empleado por la clase baja y también por la alta) responda predominantemente al sistema casual en cuanto al uso de los clíticos, y que en este aspecto esté más conforme con el uso más generalizado en el mundo hispanohablante y, al parecer, en España misma, la clase media local muestra clara tendencia hacia por lo menos uno de los empleos referenciales típicos de Castilla —el leismo para seres vivientes. También se observa en Soria, precisamente en varones y mujeres de clase media, alguna tendencia hacia el laísmo. Pero ésta es bastante menos acusada - lo cual no es de extrañar dado que el laismo es generalmente objeto 


\section{Cundro 9}

Frecuencia de usos referenciales en Logroño en función de la clase y el sexo del hablante

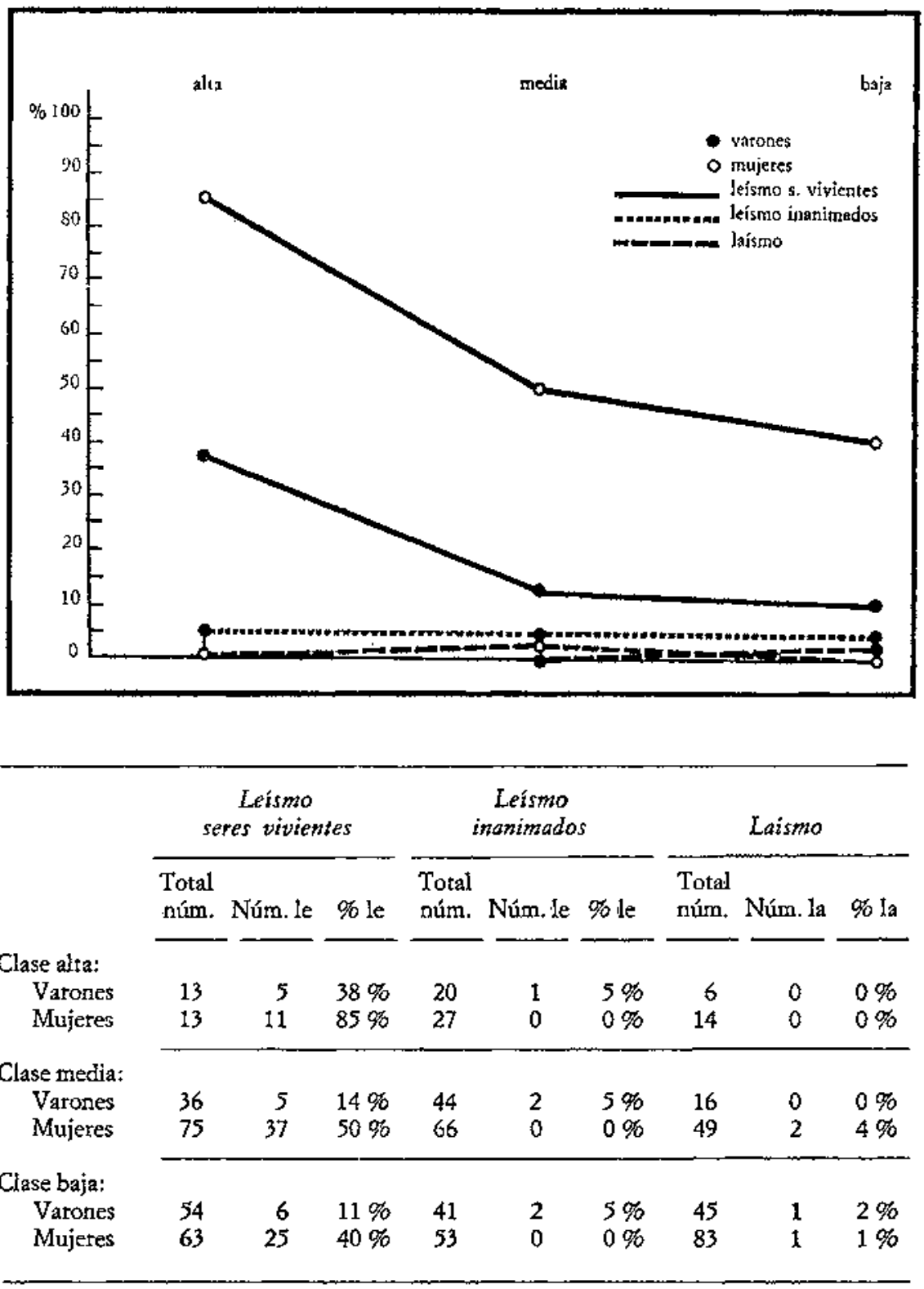


de reprobación fuera de la zona en que es nativo, y que es teprobado también por la gramática prescriptiva. ${ }^{16}$

El interés que ofrecen los resultados de Logroño también se basa en una discrepancia respecto a lo que aparece como el vernáculo nativo -el empleo casual de le, la, y lo. Pero aquí el grupo discrepante no es la clase media, sino las mujeres en general. Como las mujeres han mostrado repetidamente una especial tendencia a adoptat, en contextos de discurso relativamente «formal», precisamente aquellos rasgos que se consideran "prestigiosos» localmente, debemos concluir que el leísmo para seres vivientes constituye un rasgo de prestigio en la zona tradicionalmente casteliana en general, tanto si este empleo es rativo en la localidad en cuestión (como lo es en Valladolid) como si no to es (el caso de Soria y Logroño) ${ }^{17}$ También concuerda con esta conclusión el hecho de que, en Logroño, el leismo para seres vivientes sea el único empleo referencial de frecuencia relativamente alta en la clase alta en general, y que en Valladolid sea el único de frecuencia tan alta entre los vatones de clase alta como en los de la clase media y baja.

No obstante, la adopción de empleos referenciales en Logroño no parece comparable, cualitativamente, con la que observamos en Soria, precisamente porque en Logroño es más que nada característica de mujeres (por lo menos en los datos de que disponemos en este momento, como puede verse en la nota 11). Por lo tanto, esta tendencia "castellanizante» no parece estar entretejida con la estructura social de Logroño tal como lo está en la de Soria, donde caracteriza a la clase media en general.

Seria conveniente poder relacionar diferencias socio-lingüísticas de este tipo con las diferencias socio-económicas evidentes entre Soria y Logroño. ${ }^{18}$ En particular, sería interesante ver si Logtoño se va distanciando progtesivamente más de las otras provincias históricamente castellanas $f y$ si, por

16. Real Academia Española, Gramática de la lengua española: Nueva edición, reformada, de 1931 (Madrid: RAE, 1962), y Esbozo de una nueva gramática de la lengua española (Madrid: Espasa-Calpe, 1977). La evolución de la postura académica respecto al empleo de los clíticos (resumida en Cuervo, op. cit.) es interesante e intrigante por sus implicaciones socio-políticas, ya que en efecto representa una progresiva toma de posición a favor del uso hispánico más general y en contra del de Castilla.

17. Labov, 1972, op. cit., capítulos 8 y 9. El «prestigio» socio-linguístico es un concepto más restringido que la evaluación positiva en general, hallándose más vinculado al empleo de las clases prestigiosas, a nociones de «corrección», etc.

18. Fundación FOESSA, Intorme sociológico sobre la situación social de España (Madtid: Eutamérica, 1970); Carlos Carrasco-Muñoz de Vera, La alternativa de Castillo $y$ Lebn: Datos para una verdadera autonomia (Madrid: De la Torre, 1978). 
lo contrario, Soria se va acercando más a ellas) a partir de las recientes tomas de posición político-administrativas respecto a Castilla, aparentemente fruto de estas mismas diferencias. Para ello sería necesario tomar en consideración no sólo una muestra más fabłle sino también un mayor nú mero de variables. También conviene distinguir más variables independientes (como de hecho estamos haciendo para la versión completa de este estudio), siendo quizá la edad de los hablantes la variable social-demográfica adicional de mayor interés, por su relación más directa con la investigación del cambio en valores sociales. ${ }^{19}$

\section{FLORA KLEIN}

School of Languages and Linguistics

Georgetown University Washington DC, 20057

USA

19. También pensamos aislat la variable «movilidad social ascendente», ya que en otros casos ha demostrado ser aún más influyente que Ia clase social en sí. Véase a William Labov, «The Effect of Social Mobility on Linguistic Behavior», en S. Liebetson, comp., Explorations in Sociolinguistics (La Haya: Mouton, 1967). 